\title{
Worse Prognosis for IDH Wild-Type Diffuse Gliomas With Larger Residual Biological Tumor Burden
}

\section{Hiroyuki Tatekawa}

University of California, Los Angeles

\section{Hiroyuki Uetani}

University of California, Los Angeles

Akifumi Hagiwara

University of California, Los Angeles

Shadfar Bahri

University of California, Los Angeles

\section{Catalina Raymond}

University of California, Los Angeles

\section{Albert Lai}

University of California, Los Angeles

Timothy Cloughesy

University of California, Los Angeles

\section{Phioanh Nghiemphu}

University of California, Los Angeles

Linda Liau

University of California, Los Angeles

Whitney Pope

University of California, Los Angeles

\section{Noriko Salamon}

University of California, Los Angeles

Benjamin Ellingson ( $\sim$ bellingson@mednet.ucla.edu )

University of California, Los Angeles

\section{Research Article}

Keywords: OS, IDH, FLAIR, WHO

Posted Date: January 22nd, 2021

DOI: https://doi.org/10.21203/rs.3.rs-148651/v1 
License: (c) (i) This work is licensed under a Creative Commons Attribution 4.0 International License. Read Full License 


\section{Abstract}

This study aimed to assess the association between biological tumor burden in pre- and post-operative status and overall survival (OS) in isocitrate dehydrogenase (IDH) wild-type gliomas, and to evaluate which volume was the best predictor of OS. Thirty-four patients with treatment-naïve IDH wild-type gliomas (grade II, 6; III, 15; IV, 13) were retrospectively included. Three pre-operative tumor regions of interest (ROIs) were segmented based on the contrast-enhanced (CE), fluid-attenuated inversion recovery (FLAIR) hyperintense, and 3,4-dihydroxy-6-[ $\left.{ }^{18} \mathrm{~F}\right]$-fluoro-L-phenylalanine (FDOPA) hypermetabolic regions. Resected ROls were segmented from the post-operative images. Residual CE, FLAIR hyperintense, and FDOPA hypermetabolic ROIs were created by subtracting resected ROIs from pre-operative ROIs. Cox regression was conducted to investigate the association of OS with the volume of each ROI. Residual CE volume had a significant association with $\mathrm{OS}$ (hazard ratio $[\mathrm{HR}]=1.26, P=0.039$ ), but this effect disappeared when controlling for tumor grade. Residual FDOPA hypermetabolic volume was significantly associated with OS ( $H R=1.18, P=0.008)$, even when controlling for tumor grade. FLAIR hyperintense volume showed no significant association with OS. Residual FDOPA hypermetabolic burden predicted OS for IDH wild-type gliomas, regardless of tumor grade. Furthermore, removing hypermetabolic and CE regions may improve the prognosis.

\section{Introduction}

In 2016, the World Health Organization (WHO) classification of Tumors of the Central Nervous System reclassified gliomas by integrating molecular status, such as isocitrate dehydrogenase (IDH) gene mutation and chromosomal $1 p / 19 q$ co-deletion ${ }^{1}$. Overall, approximately $90 \%$ of glioblastomas are IDH wild-type, whereas the remaining $10 \%$ are IDH mutant ${ }^{2}$. About $30 \%$ of grade II and III gliomas are IDH wild-type, whereas the remaining $70 \%$ are IDH mutant ${ }^{3,4}$. Although treatment methods vary depending on patient prognostic factors, including histology, tumor grade, age at diagnosis, Karnofsky performance status, first presenting symptom, extent of resection, and tumor size and location, as well as the molecular status, the standard treatment for patients with newly diagnosed high-grade gliomas remains surgical resection followed by radiotherapy in combination with the DNA-alkylating agent temozolomide 3 .

Regardless of tumor grade, patients with IDH wild-type gliomas present with a median overall survival (OS) $<2$ years, which is significantly shorter than that of IDH mutant gliomas ${ }^{4}$. The extent of surgical resection of contrast-enhanced (CE) regions on T1-weighted magnetic resonance imaging (MRI) has been associated with longer survival ${ }^{5}$, and a more aggressive resection beyond CE regions was recently suggested for a better prognosis ${ }^{6}$. On the other hand, amino acid positron emission tomography (PET), including 3,4-dihydroxy-6-[ $\left[{ }^{18} \mathrm{~F}\right]$-fluoro-L-phenylalanine (FDOPA) and O-(2-[18 F]-fluoroethyl)-L-tyrosine (FET), provides metabolic information to complement MRI-derived information. Several PET imaging metrics, including standard uptake value (SUV) and hypermetabolic volume (referred to as biological tumor volume [BTV]), were investigated to predict prognosis for gliomas. The BTV for glioblastomas with post- 
operative pre-radio-chemotherapy and for recurrent high-grade gliomas were reported to be a significant predictor of $\mathrm{OS}^{7-11}$.

To date, no studies have evaluated the association of tumor burden, including CE volume, fluidattenuated inversion recovery (FLAIR) hyperintense volume, and BTV, with OS focusing exclusively on IDH wild-type gliomas, although patients with IDH wild-type gliomas are known to have a poor prognosis, regardless of tumor grade. The purpose of the current study was to assess whether the tumor burden, including CE, FLAIR hyperintense, and FDOPA hypermetabolic regions, in pre- and post-operative exams are associated with OS in patients with IDH wild-type gliomas. We hypothesize that hypermetabolic tumor burden may be a strong predictor of OS compared with volume of CE or FLAIR hyperintense regions.

\section{Results}

The current study included 34 treatment-naïve IDH wild-type glioma patients (13 females) with a mean age \pm standard deviation of $61.0 \pm 10.8$ years at the time of the PET examination. Nine patients underwent partial resection/biopsy, 19 underwent subtotal resection, and 6 underwent gross total resection. According to the 2007/2016 WHO criteria, 6 gliomas were grade II, 15 were grade III, and 13 were grade IV. Detailed patient demographics are shown in Tables 1 and 2. Seventeen patients did not have CE regions on pre-operative images (grade II, 5; grade III, 10; grade IV, 2), while six patients did not have FDOPA hypermetabolic regions (grade II, 2; grade III, 3; grade IV, 1).

Cox univariate analysis (Table 3 ) showed significant associations of OS with the residual CE volume (hazard ratio $[\mathrm{HR}]=1.26,95 \%$ confidence interval $[\mathrm{Cl}]=1.01-1.57 \mathrm{P}=0.039$ ) and residual FDOPA hypermetabolic volume $(\mathrm{HR}=1.18,95 \% \mathrm{Cl}=1.04-1.33, \mathrm{P}=0.008)$. The Akaike information criterion (AIC) was the lowest for residual FDOPA hypermetabolic volume (AIC = 87.15), indicating that residual FDOPA hypermetabolic volume is the best predictor of OS. These associations remained significant when controlling for age (residual $\mathrm{CE}$ volume: $\mathrm{HR}=1.26,95 \% \mathrm{Cl}=1.01-1.56, \mathrm{P}=0.037, \mathrm{AIC}=91.94$; residual FDOPA hypermetabolic volume: $\mathrm{HR}=1.21,95 \% \mathrm{Cl}=1.06-1.37, \mathrm{P}=0.004, \mathrm{AIC}=88.17$ ). Only residual FDOPA hypermetabolic volume showed significant associations when controlling for tumor grade (residual $\mathrm{CE}$ volume: $\mathrm{HR}=1.2,95 \% \mathrm{Cl}=0.92-1.55, \mathrm{P}=0.18$, $\mathrm{AIC}=91.25$; residual FDOPA hypermetabolic volume: $\mathrm{HR}=1.16,95 \% \mathrm{Cl}=1.01-1.34, \mathrm{P}=0.044, \mathrm{AIC}=88.97$ ) or controlling for age and tumor grade (residual $\mathrm{CE}$ volume: $\mathrm{HR}=1.19,95 \% \mathrm{Cl}=0.93-1.53, \mathrm{P}=0.17, \mathrm{AIC}=93.12$; residual FDOPA hypermetabolic volume: $\mathrm{HR}=1.19,95 \% \mathrm{Cl}=1.02-1.37, \mathrm{P}=0.023, \mathrm{AIC}=89.95)$. Even when tumor grade was treated as a binary variable (WHO grade II vs. III and IV, or WHO grade II and III vs. IV), the significances did not affect the ability to predict OS. The AIC remained lower for residual FDOPA hypermetabolic volume than residual CE volume when controlling for clinical information, indicating that residual FDOPA hypermetabolic volume is a better predictor of OS.

\section{Discussion}


This study evaluated whether the tumor burden, including pre- or post-operative CE, FLAIR hyperintensity, or FDOPA hypermetabolic volume, was associated with OS in IDH wild-type gliomas. Residual FDOPA hypermetabolic volume after surgery was the strongest predictor of OS when controlling for age and tumor grade.

Previous studies using FET PET showed that BTV before radio-chemotherapy was a significant predictor of OS for glioblastoma, but did not evaluate whether this applied to other tumor grades ${ }^{7,8}$. Another study using ${ }^{11} \mathrm{C}$-methionine PET also described BTV as a significant predictor of OS for recurrent gliomas of grade III/IV ${ }^{9}$. No studies, to the best our knowledge, have yet evaluated the association of OS with BTV specifically within IDH wild-type gliomas. This study revealed that residual BTV after surgery was significantly associated with OS for IDH wild-type gliomas regardless of tumor grade. Since most newly diagnosed glioblastomas are biologically IDH wild-type ${ }^{2}$, our results are consistent with the findings of previous studies evaluating higher grade gliomas using amino acid PET ${ }^{7,8}$. Coversely, due to the biological similarities to glioblastomas ${ }^{12}$, patients with lower-grade IDH wild-type gliomas also showed shorter survival than patients with IDH mutant gliomas. As such, residual BTV could be a crucial biomarker to predict prognosis for IDH wild-type gliomas regardless of tumor grade.

This study suggests that CE volume may not be an appropriate biomarker to predict prognosis especially for lower-grade gliomas with IDH wild-type due to the relatively low rate of contrast enhancement. Several previous studies have documented an association of OS with residual CE volume on post-operative images for glioblastomas ${ }^{5,13}$. However, the residual CE volume, which showed a significant association with OS in Cox univariate regression analysis, was not significantly associated with OS when controlling for tumor grade. A small population size may have influenced the statistical power. Meanwhile, $71 \%$ $(15 / 21)$ of grade II/III IDH wild-type gliomas showed no contrast enhancement, and 15\% (2/13) of grade IV glioblastomas showed no contrast enhancement in this study. Less than half of grade II/III IDH wildtype gliomas are reported to show contrast enhancement 4,14 , and not all grade IV glioblastomas showed contrast enhancement. Therefore, CE volume should be paid particular attention in order to best predict prognosis in lower-grade IDH wild-type gliomas.

The gross macroscopic resection of the $\mathrm{CE}$ component of glioblastomas was associated with longer survival ${ }^{13,15}$. A recent large cohort study supported this association ${ }^{2}$. MRI is the first choice for treatment planning, and the maximal resection of CE regions represents the neurosurgical standard, when safely feasible. A more aggressive resection of FLAIR hyperintense regions was also suggested ${ }^{6}$. However, contrast enhancement is seen in areas with disrupted blood-brain barrier; additionally, FLAIR hyperintense regions can reflect tissue edema, and thereby do not necessarily correspond to the extent of tumor cells. Furthermore, due to the inherent trade-off between the extent of the resection of the tumor region and postoperative functional status, aggressive regression requires sufficient attention. Meanwhile, amino acid tracer accumulates in tumor cells and could define tumor extension more accurately, especially in metabolically active non-CE FLAIR hyperintense regions ${ }^{16-18}$. Our results suggest that maximal surgical reduction of the BTV may contribute to the better prognosis of patients with resectable gliomas, and that 
residual hypermetabolic volume is a more useful biomarker to predict prognosis than CE volume. Therefore, the maximal removal of both hypermetabolic and CE regions may improve the prognosis of patients with IDH wild-type gliomas with relatively small functional defects.

There are a number of limitations to our study. First, for gliomas with large necrotic regions or mass effects, the resected cavity may shrink or move from pre-surgical locations after surgery, incurring difficulties in segmentation. To mitigate this problem, two neuroradiologists validated the resected regions-of-interest (ROIs) by consensus using all available images. Alternatively, we calculated all imaging metrics from pre-operative images; thus, the strength of our method lies in the fact that imaging metrics do not suffer from post-surgical effects, such as blood-brain barrier breakdown or a postoperative flare phenomenon, which may incur additional contrast enhancement or amino acid tracer uptake ${ }^{19}$. Second, due to the retrospective nature of the study, the parameters of MRI sequences and the term between PET examination and surgery or between surgery and post-operative imaging examination were not consistent. In particular, the time period between surgery and post-operative imaging is associated with post-surgical contrast enhancement or edema, which may affect the segmentation of resected ROls 20,21 . The third limitation is the small population size. A larger study with an external dataset to validate the association between the extent of resection of hypermetabolic regions is warranted. Fourth, additional clinical information, including Karnofsky performance status and clinical symptoms, and tumor markers, including O(6)-methylguanine-DNA methyltransferase (MGMT) methylation and epidermal growth factor receptor (EGFR) mutation/amplification, are important to evaluate survival; however, they were not available for all subjects, and were not analyzed in this study. Furthermore, this study did not take postoperative treatment methods nor tumor location into account, although these factors may affect prognosis.

In conclusion, residual FDOPA hypermetabolic tumor burden after surgery was significantly associated with OS for IDH wild-type gliomas, even when controlling for age and tumor grade. In the context of surgical planning, the maximal removal of hypermetabolic regions as well as CE regions may improve the prognosis of patients with IDH wild-type gliomas.

\section{Methods}

\section{Patient Selection}

A total of 37 patients with treatment naïve and histologically confirmed gliomas who underwent preoperative FDOPA PET and MRI scans between 2007 and 2020 were retrospectively selected. MRI scans were performed within 2 months of the corresponding PET scans. All patients were diagnosed with IDH wild-type gliomas based on IDH1 mutational status. No patients underwent stereotactic biopsy prior to FDOPA PET or MRI scans. Among them, one patient was excluded from this study because the period between their FDOPA PET and surgery exceeded half a year, and two patients were excluded because they did not undergo a follow-up MRI examination after surgery. Finally, 34 patients with IDH wild-type glioma were included in this study. OS was measured from the time of the PET scan until death or the censored 
date (median time, 617 days; range, 29-2241). The median time between PET scan and surgery was 18 days (range, 1-178 days), while the median time between surgery and the first post-operative MRI was 18 days (range, $0-101$ days).

This retrospective study was approved by the "Medical IRB \#2" at the University of California Los Angeles. All procedures performed in studies involving human participants were in accordance with the ethical standards of the institutional and/or national research committee and with the 1964 Helsinki declaration and its later amendments or comparable ethical standards. Written informed consent was obtained from all individual participants to have their advanced imaging, clinical, and molecular data included in our IRB-approved research database according to IRB\#10-000655. Some subjects were also included in a previous study ${ }^{22}$, which evaluated voxel-wise imaging correlations between FDOPA and MRI.

\section{FDOPA PET Image Acquisition}

A full-ring PET/computed tomography (CT) scanner (ECAT-HR; Siemens, Knoxville, TN, USA) was used to obtain PET images after the subjects fasted for more than four hours. A CT was performed before the PET scan for attenuation correction. FDOPA was synthesized and injected intravenously, following previously reported procedures ${ }^{23,24}$. Three-dimensional FDOPA emission data were obtained for 30 minutes, and the data were integrated between 10 and 30 minutes following the injection to obtain 20minute static FDOPA images after reconstruction. FDOPA PET images were reconstructed using an ordered-subset expectation maximization iterative reconstruction algorithm with six iterations and eight subsets ${ }^{25,26}$. Then, a Gaussian filter with a full width at half maximum of $4 \mathrm{~mm}$ was applied. The resulting voxel sizes were $1.34 \times 1.34 \times 3 \mathrm{~mm}$ for FDOPA PET images. SUV maps of FDOPA were calculated based on the radioactive activity divided by the decay-corrected injected dose per body mass. Resulting SUV maps were subsequently normalized (nSUV) relative to the median value of the normalappearing striatum ${ }^{22,27}$.

\section{Magnetic Resonance Image Acquisition}

The anatomical MRI scans consisted of standard T1-weighted pre- and post-contrast images (2D axial turbo spin echo with a 3-mm slice thickness and no interslice gap, or 3D inversion-prepared gradient echo images with 1-1.5 mm isotropic voxel size) ${ }^{28}$. The T2-weighted FLAIR images were acquired with a 3$\mathrm{mm}$ slice thickness and no interslice gap using a 1.5-T or 3-T clinical MRI scanner. Anatomical images were obtained after surgery (closest date to the surgery), as well as before surgery.

\section{Postprocessing and ROI Analysis}

Pre-operative MRI and PET images were registered to the pre-operative post-contrast T1-weighted images for each patient using a rigid transformation with 6-degree of freedom and a mutual information cost function using FMRIB Software Library (FSL) software (flirt; FMRIB, Oxford, UK; http://www.fmrib.ox.ac.uk/fsl/). Post-operative MRI scans were also registered to the post-operative post- 
contrast T1-weighted images, and then registered to the pre-operative post-contrast T1-weighted images using a rigid transformation with 6-degree of freedom and a mutual information cost function.

Figure 1 illustrates the process of $\mathrm{ROI}$ analysis. Using pre-operative images, three tumor ROls were segmented based on (1) the CE regions on post-contrast T1-weighted images, (2) the hyperintense regions on T2-weighted FLAIR images, and (3) the FDOPA hypermetabolic regions on PET images by a board-certificated neuroradiologist (H.T. with 12 years of clinical experience) using Analysis of Functional Neurolmages software (AFNI; NIMH Scientific and Statistical Computing Core; Bethesda, MD, USA; https://afni.nimh.nih.gov). For the segmentation of CE and FLAIR ROIs, a semi-automatic method was employed in which a large ROI was drawn over a CE or FLAIR hyperintensity region ${ }^{29}$. Intensity thresholds were then chosen on each patient to extract only CE regions and hyperintense regions from the post-contrast T1-weighted and FLAIR images, respectively. For FLAIR hyperintense ROIs, necrotic areas were not excluded if they showed hyperintensity on FLAIR images. FDOPA hypermetabolic ROls within FLAIR hyperintense ROls were extracted with a higher threshold than the median uptake value of the striatum.

Using post-operative images, which were registered to pre-operative images, a single ROI corresponding to the resected tumor was segmented based on the cavity of removed lesions. For gliomas with a large necrotic region or mass effect, the segmentation of resected ROls was complicated since the cavity may have moved from the pre-surgical regions. The resected ROls were, therefore, determined by using all available pre- and post-operative images, including CE-T1WI, non-CE-T1WI, T2WI, and FLAIR images, to differentiate the resected ROIs from adjacent brain regions by referring to normal brain structures as well as resected cavities. Then, the ROls were validated by another board-certificated neuroradiologist (H.U. with 14 years of clinical experience) by consensus.

Finally, the registered resected ROIs were subtracted from the CE ROIs, FLAIR ROIs, and FDOPA hypermetabolic ROIs, resulting in residual CE ROIs, residual FLAIR ROIs, and residual FDOPA hypermetabolic ROIs, respectively. All volumes of tumor ROls are reported in milliliters $(\mathrm{mL})$. The maximum nSUV ( nSUV $_{\text {max }}$ ) within the FLAIR ROIs and residual FLAIR ROIs was also calculated.

\section{Statistical Analyses}

Cox univariate proportional hazards regression analyses were conducted to investigate the association between OS and predictor variables including clinical information, such as age and tumor grade, and imaging metrics, such as the CE volume, residual CE volume, FLAIR volume, residual FLAIR volume, FDOPA hypermetabolic volume, residual FDOPA hypermetabolic volume, $\mathrm{nSUV}_{\text {max }}$, and residual $\mathrm{nSUV}_{\text {max }}$. All variables, including tumor grade, were treated as continuous variables. When the CE or FDOPA hypermetabolic regions were unidentifiable or completely resected, the volumes represented zero $\mathrm{mL}$. For imaging metrics showing a significant association in the Cox univariate regression, Cox multivariate regression analyses controlling for age, tumor grade, or both age and tumor grade were performed separately. To compare the fitness of the regression model, the AIC was calculated. The most fitted model 
is the one with the minimum AIC among all models. Statistical analysis was performed using R software (version 3.5.2; http://www.r-project.org/), and statistical significance was defined at $\mathrm{P}<0.05$.

\section{Declarations}

\section{Data availability}

Data involved in this study, may be made available on request form the corresponding author (B.M.E.). Patient imaging data are not publicly available due privacy restrictions, i.e. presence of personal health information.

\section{Authors' contributions}

Study design: H.T., H.U., A.H., S.B., N.S., B.M.E. Acquisition: A.L., T.F.C, P.L.N., L.M.L. Image and data analysis: H.T., H.U., A.H., C.R., W.B.P., N.S., B.M.E. The first draft of the manuscript was written by H.T and all authors commented on previous versions of the manuscript. All authors read and approved the final manuscript.

\section{Acknowledgements/Grant}

Grant from the Society of Nuclear Medicine and Molecular Imaging (SNMMI) (H.T.); American Cancer Society (ACS) Research Scholar Grant (RSG-15-003-01-CCE) (B.M.E.); American Brain Tumor Association (ABTA) Research Collaborators Grant (ARC1700002) (B.M.E.); National Brain Tumor Society (NBTS) Research Grant (B.M.E., T.F.C); NIH/NCI UCLA Brain Tumor SPORE (1P50CA211015-01A1) (B.M.E., L.A., T.F.C, P.L.N); NIH/NCl (1R21CA223757-01) (B.M.E.)

\section{Additional information}

Competing interests: B.M.E.-Advisory Board- Hoffman La-Roche; Siemens; Nativis; Medicenna; MedQIA; Bristol-Myers Squibb; Imaging Endpoints; Agios. Paid Consultant-Nativis; MedQIA; Siemens; Hoffman La-Roche; Imaging Endpoints; Medicenna; Agios. Grant Funding-Hoffman La-Roche; Siemens; Agios; Janssen. B.M.E. also holds a patent on this technology (US Patent \#15/577,664; International PCT/US2016/034886). T.F.C.-Advisory Board-Roche/ Genentech, Amgen, Tocagen, NewGen, LPath, Proximagen, Celgene, Vascular Biogenics Ltd, Insys, Agios, Cortice Bioscience, Pfizer, Human Longevity, BMS, Merck, Notable Lab, MedQIA. The other authors (H.T., H.U., A.H., S.B., C.R., A.L, P.L.N., L.M.L., W.B.P, N.S.) declare no competing interests.

\section{References}

1. Louis, D. N. et al. The 2016 World Health Organization Classification of Tumors of the Central Nervous System: a summary. Acta Neuropathol. 131, 803-820 https://doi.org/10.1007/s00401-0161545-1 (2016). 
2. Molinaro, A. M. et al. Association of Maximal Extent of Resection of Contrast-Enhanced and NonContrast-Enhanced Tumor With Survival Within Molecular Subgroups of Patients With Newly Diagnosed Glioblastoma. JAMA Oncol. https://doi.org/10.1001/jamaoncol.2019.6143 (2020).

3. Gittleman, H., Sloan, A. E. \& Barnholtz-Sloan, J. S. An independently validated survival nomogram for lower grade glioma. Neuro Oncol. https://doi.org/10.1093/neuonc/noz191 (2019).

4. Maynard, J. et al. World Health Organization Grade II/III Glioma Molecular Status: Prediction by MRI Morphologic Features and Apparent Diffusion Coefficient. Radiology. 191832, https://doi.org/10.1148/radiol.2020191832 (2020).

5. Ellingson, B. M., Wen, P. Y. \& Cloughesy, T. F. Evidence and context of use for contrast enhancement as a surrogate of disease burden and treatment response in malignant glioma. Neuro Oncol. 20, 457-471 https://doi.org/10.1093/neuonc/nox193 (2018).

6. Lasocki, A., Gaillard, F., Non-Contrast-Enhancing \& Tumor A New Frontier in Glioblastoma Research. AJNR Am J Neuroradiol. 40, 758-765 https://doi.org/10.3174/ajnr.A6025 (2019).

7. Suchorska, B. et al. Biological tumor volume in 18FET-PET before radiochemotherapy correlates with survival in GBM. Neurology. 84, 710-719 https://doi.org/10.1212/wnl.0000000000001262 (2015).

8. Poulsen, S. H. et al. The prognostic value of FET PET at radiotherapy planning in newly diagnosed glioblastoma. Eur J Nucl Med Mol Imaging. 44, 373-381 https://doi.org/10.1007/s00259-016-34942 (2017).

9. Jung, T. Y. et al. Prognostic value of post-treatment metabolic tumor volume from (11)C-methionine PET/CT in recurrent malignant glioma. Neurosurg Rev. 40, 223-229 https://doi.org/10.1007/s10143016-0748-1 (2017).

10. Piroth, M. D. et al. Prognostic impact of postoperative, pre-irradiation (18)F-fluoroethyl--tyrosine uptake in glioblastoma patients treated with radiochemotherapy. Radiother Oncol. 99, 218-224 https://doi.org/10.1016/j.radonc.2011.03.006 (2011).

11. Pirotte, B. J. et al. Positron emission tomography-guided volumetric resection of supratentorial highgrade gliomas: a survival analysis in 66 consecutive patients. Neurosurgery. 64, 471-481 discussion 481 https://doi.org/10.1227/01.NEU.0000338949.94496.85 (2009).

12. Olar, A. et al. IDH mutation status and role of WHO grade and mitotic index in overall survival in grade II-III diffuse gliomas. Acta Neuropathol. 129, 585-596 https://doi.org/10.1007/s00401-0151398-z (2015).

13. Grabowski, M. M. et al. Residual tumor volume versus extent of resection: predictors of survival after surgery for glioblastoma. J Neurosurg. 121, 1115-1123 https://doi.org/10.3171/2014.7.JNS132449 (2014).

14. Suchorska, B. et al. Contrast enhancement is a prognostic factor in IDH1/2 mutant, but not in wildtype WHO grade II/III glioma as confirmed by machine learning. Eur J Cancer. 107, 15-27 https://doi.org/10.1016/j.ejca.2018.10.019 (2019).

15. Hardesty, D. A. \& Sanai, N. The value of glioma extent of resection in the modern neurosurgical era. Front Neurol. 3, 140 https://doi.org/10.3389/fneur.2012.00140 (2012). 
16. Suchorska, B. et al. (18)F-FET-PET as a biomarker for therapy response in non-contrast enhancing glioma following chemotherapy. J Neurooncol. 139, 721-730 https://doi.org/10.1007/s11060-0182919-0 (2018).

17. Kosztyla, R. et al. High-grade glioma radiation therapy target volumes and patterns of failure obtained from magnetic resonance imaging and 18F-FDOPA positron emission tomography delineations from multiple observers. Int J Radiat Oncol Biol Phys. 87, 1100-1106 https://doi.org/10.1016/j.jijobp.2013.09.008 (2013).

18. Kobayashi, K. et al. Prognostic value of volume-based measurements on (11)C-methionine PET in glioma patients. Eur J Nucl Med Mol Imaging. 42, 1071-1080 https://doi.org/10.1007/s00259-0153046-1 (2015).

19. Filss, C. P. et al. Flare Phenomenon in O-(2-(18)F-Fluoroethyl)-I-Tyrosine PET After Resection of Gliomas. J Nucl Med. 61, 1294-1299 https://doi.org/10.2967/jnumed.119.238568 (2020).

20. Albert, F. K., Forsting, M., Sartor, K., Adams, H. P. \& Kunze, S. Early postoperative magnetic resonance imaging after resection of malignant glioma: objective evaluation of residual tumor and its influence on regrowth and prognosis. Neurosurgery. 34, 45-60 discussion $60-41$ https://doi.org/10.1097/00006123-199401000-00008 (1994).

21. Majos, C. et al. Early post-operative magnetic resonance imaging in glioblastoma: correlation among radiological findings and overall survival in 60 patients. Eur Radiol. 26, 1048-1055 https://doi.org/10.1007/s00330-015-3914-x (2016).

22. Tatekawa, H. et al. Voxel-Wise and Patient-Wise Correlation of (18)F-FDOPA PET, rCBV, and ADC in Treatment-Naïve Diffuse Gliomas with Different Molecular Subtypes. J Nucl Med. https://doi.org/10.2967/jnumed.120.247411 (2020).

23. Bishop, A. et al. Proton irradiation of [180]02: production of [18F]F2 and [18F]F2 + [18F] OF2. Nucl Med Biol. 23, 189-199 https://doi.org/10.1016/0969-8051(95)02037-3 (1996).

24. Namavari, M., Bishop, A., Satyamurthy, N., Bida, G. \& Barrio, J. R. Regioselective radiofluorodestannylation with [18F]F2 and [18F]CH3COOF: a high yield synthesis of 6-[18F]Fluoro-Ldopa. Int J Rad App/ Instrum A. 43, 989-996 https://doi.org/10.1016/0883-2889(92)90217-3 (1992).

25. Kinahan, P. E., Townsend, D. W., Beyer, T. \& Sashin, D. Attenuation correction for a combined 3D PET/CT scanner. Med Phys. 25, 2046-2053 https://doi.org/10.1118/1.598392 (1998).

26. Nuyts, J., Michel, C. \& Dupont, P. Maximum-likelihood expectation-maximization reconstruction of sinograms with arbitrary noise distribution using NEC-transformations. IEEE Trans Med Imaging. 20, 365-375 https://doi.org/10.1109/42.925290 (2001).

27. Oughourlian, T. C. et al. Rate of change in maximum (18)F-FDOPA PET uptake and non-enhancing tumor volume predict malignant transformation and overall survival in low-grade gliomas. $J$ Neurooncol. 147, 135-145 https://doi.org/10.1007/s11060-020-03407-w (2020).

28. Ellingson, B. M. et al. Consensus recommendations for a standardized Brain Tumor Imaging Protocol in clinical trials. Neuro Oncol. 17, 1188-1198 https://doi.org/10.1093/neuonc/nov095 (2015). 
29. Ellingson, B. M. et al. Recurrent glioblastoma treated with bevacizumab: contrast-enhanced T1weighted subtraction maps improve tumor delineation and aid prediction of survival in a multicenter clinical trial. Radiology. 271, 200-210 https://doi.org/10.1148/radiol.13131305 (2014).

\section{Tables}

Table 1 Patient demographics and imaging metrics for IDH wild-type gliomas Number of patients 34

Female $13(38 \%)$

Age \pm SD (year) $61.0 \pm 10.8$

WHO classification grade II $6(18 \%)$ III 15 (44\%) IV $13(38 \%)$

Surgical procedure Biopsy/partial resection $9(26 \%)$ Subtotal resection 19 (56\%)

Gross total resection

$6(18 \%)$

Contrast enhanced volume $\pm \mathrm{SD}(\mathrm{mL})$ $1.31 \pm 2.84$

Residual contrast enhanced volume $\pm \mathrm{SD}(\mathrm{mL})$

$0.78 \pm 2.13$

FLAIR hyperintense volume \pm SD $(\mathrm{mL})$ $44.90 \pm 47.99$

Residual FLAIR hyperintense volume \pm SD $(\mathrm{mL})$ $38.05 \pm 44.51$

FDOPA hypermetabolic volume \pm SD $(\mathrm{mL})$ $4.63 \pm 5.53$

Residual FDOPA hypermetabolic volume \pm SD $(\mathrm{mL})$

$2.77 \pm 3.80$

$\mathrm{nSUV}_{\max } \pm \mathrm{SD}$

$1.65 \pm 0.75$

Residual $\mathrm{nSUV}_{\max } \pm \mathrm{SD}$ $1.58 \pm 0.68$

$\mathrm{SD}=$ standard deviation; IDH = isocitrate dehydrogenase; $\mathrm{nSUV}_{\max }=$ maximum normalized standard uptake value; $\mathrm{WHO}=$ World Health Organization; FLAIR = fluid-attenuated inversion recovery; FDOPA = 3,4-dihydroxy-6-[18F]-fluoro-L-phenylalanine 


\begin{tabular}{|c|c|c|c|c|c|c|}
\hline $\begin{array}{l}\text { Patient } \\
\text { ID }\end{array}$ & Sex & Age & $\begin{array}{l}\text { WHO } \\
\text { grade }\end{array}$ & $\begin{array}{l}\text { Surgical } \\
\text { procedure }\end{array}$ & $\begin{array}{l}\text { Date between PET scan and } \\
\text { censored date (day) }\end{array}$ & $\begin{array}{l}\text { Final } \\
\text { status }\end{array}$ \\
\hline 1 & Female & 63 & $\|$ & Biopsy/PR & 2241 & Deceased \\
\hline 2 & Male & 63 & II & STR & 763 & LFU \\
\hline 3 & Male & 61 & II & STR & 1441 & Deceased \\
\hline 4 & Male & 64 & II & Biopsy/PR & 344 & Alive \\
\hline 5 & Female & 50 & II & GTR & 95 & Alive \\
\hline 6 & Male & 69 & II & STR & 388 & Alive \\
\hline 7 & Male & 63 & III & Biopsy/PR & 70 & LFU \\
\hline 8 & Female & 63 & III & Biopsy/PR & 490 & LFU \\
\hline 9 & Female & 60 & III & Biopsy/PR & 377 & Deceased \\
\hline 10 & Male & 68 & III & GTR & 1421 & LFU \\
\hline 11 & Female & 60 & III & STR & 771 & Deceased \\
\hline 12 & Male & 60 & III & STR & 771 & Deceased \\
\hline 13 & Male & 48 & III & STR & 504 & Deceased \\
\hline 14 & Female & 59 & III & STR & 730 & Deceased \\
\hline 15 & Female & 61 & III & STR & 1351 & LFU \\
\hline 16 & Male & 58 & III & STR & 857 & Deceased \\
\hline 17 & Male & 53 & III & STR & 391 & LFU \\
\hline 18 & Male & 59 & III & STR & 1014 & Deceased \\
\hline 19 & Female & 68 & III & STR & 39 & Deceased \\
\hline 20 & Male & 62 & III & STR & 226 & LFU \\
\hline 21 & Male & 64 & III & GTR & 287 & Alive \\
\hline 22 & Male & 76 & IV & Biopsy/PR & 78 & Deceased \\
\hline 23 & Female & 65 & IV & STR & 854 & Alive \\
\hline 24 & Female & 47 & IV & STR & 1675 & Deceased \\
\hline 25 & Female & 26 & IV & Biopsy/PR & 745 & Deceased \\
\hline 26 & Male & 64 & IV & GTR & 1770 & Deceased \\
\hline 27 & Male & 69 & IV & Biopsy/PR & 962 & Deceased \\
\hline
\end{tabular}




\begin{tabular}{|lllllll|}
\hline 28 & Male & 77 & IV & STR & 729 & Deceased \\
\hline 29 & Male & 74 & IV & Biopsy/PR & 39 & Deceased \\
\hline 30 & Female & 55 & IV & STR & 828 & LFU \\
\hline 31 & Male & 74 & IV & GTR & 29 & LFU \\
\hline 32 & Male & 36 & IV & STR & 404 & Deceased \\
\hline 33 & Male & 56 & IV & STR & 217 & LFU \\
\hline 34 & Female & 76 & IV & GTR & 189 & Deceased \\
\hline $\begin{array}{l}\text { IDH = isocitrate dehydrogenase; WHO = World Health Organization; PR = partial resection; STR }= \\
\text { subtotal resection; GTR = gross total resection; LFU = lost to follow-up }\end{array}$ & \\
\hline
\end{tabular}

Table 3 Cox univariate regression and Akaike information criterion

\begin{tabular}{|c|c|c|c|c|}
\hline & $\begin{array}{l}\text { Hazard } \\
\text { ratio }\end{array}$ & $\begin{array}{l}95 \% \text { confidential } \\
\text { interval }\end{array}$ & $\begin{array}{l}P \\
\text { value }\end{array}$ & AIC \\
\hline Age (year) & 1.00 & $0.95-1.06$ & 0.99 & 93.18 \\
\hline Tumor grade & 1.66 & $0.85-3.23$ & 0.14 & 90.75 \\
\hline Contrast enhanced volume (mL) & 1.05 & $0.90-1.22$ & 0.56 & 92.86 \\
\hline $\begin{array}{l}\text { Residual contrast enhanced volume } \\
(\mathrm{mL})\end{array}$ & 1.26 & $1.01-1.57$ & $0.039 *$ & 89.98 \\
\hline FLAIR hyperintense volume (mL) & 1.01 & $0.99-1.03$ & 0.21 & 91.75 \\
\hline $\begin{array}{l}\text { Residual FLAIR hyperintense volume } \\
(\mathrm{mL})\end{array}$ & 1.01 & $0.99-1.03$ & 0.30 & 92.21 \\
\hline FDOPA hypermetabolic volume (mL) & 1.05 & $0.98-1.13$ & 0.14 & 91.24 \\
\hline $\begin{array}{l}\text { Residual FDOPA hypermetabolic volume } \\
(\mathrm{mL})\end{array}$ & 1.18 & $1.04-1.33$ & $0.008^{*}$ & $87.15+$ \\
\hline $\mathrm{nSUV}_{\max }$ & 1.06 & $0.59-1.91$ & 0.84 & 93.15 \\
\hline Residual nSUV $\max$ & 1.16 & $0.63-2.16$ & 0.64 & 92.97 \\
\hline \multicolumn{5}{|c|}{$\begin{array}{l}\text { AIC = Akaike information criterion; } \mathrm{nSUV}_{\max }=\text { maximum normalized standard uptake value; FLAIR = } \\
\text { fluid-attenuated inversion recovery; FDOPA }=3,4 \text {-dihydroxy-6-[18F]-fluoro-L-phenylalanine }\end{array}$} \\
\hline
\end{tabular}



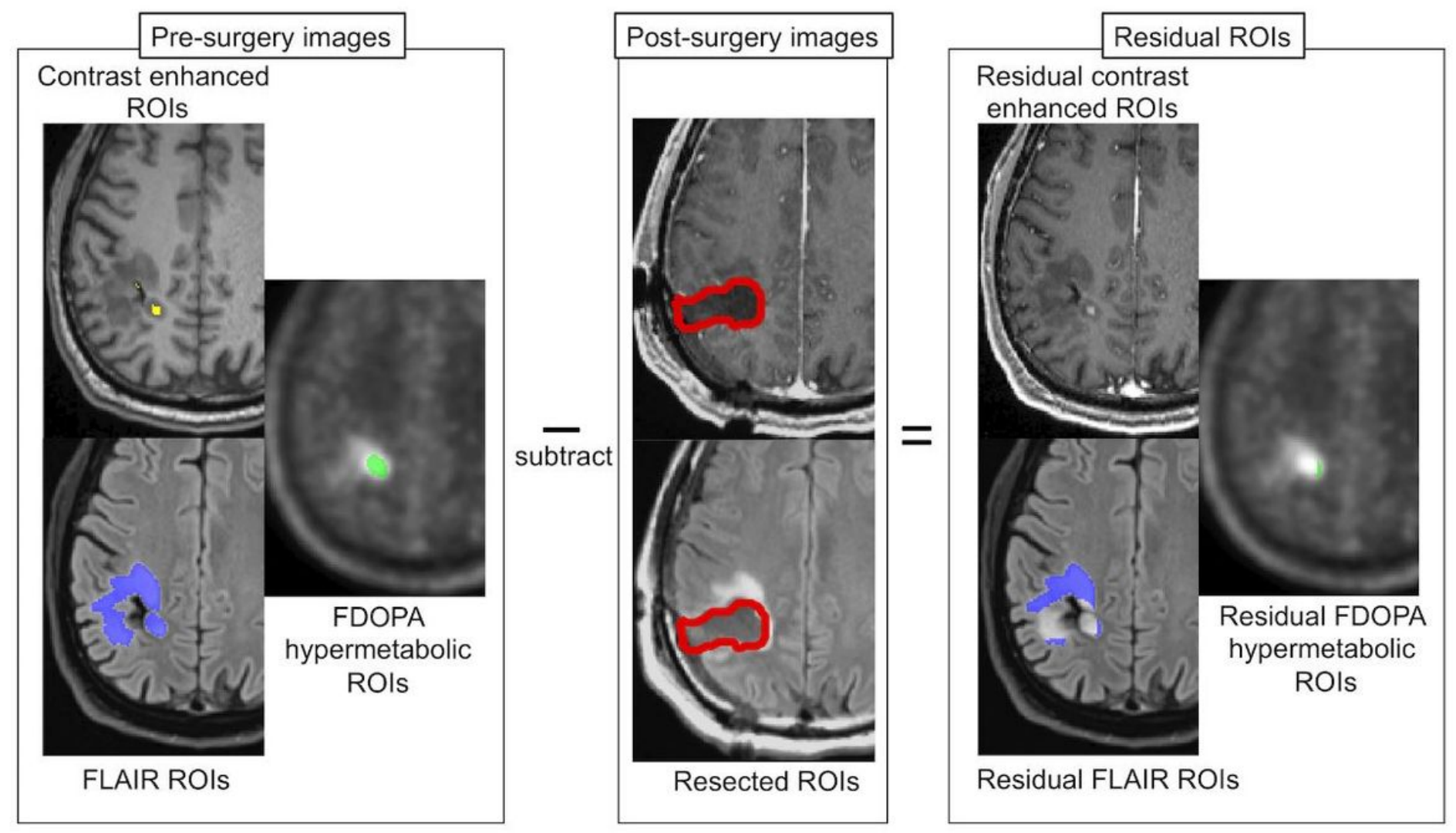

\section{Figure 1}

Processing of region-of-interest (ROI) analysis. Contrast-enhanced ROls (yellow areas), fluid-attenuated inversion recovery (FLAIR) ROIs (blue areas), and 3,4-dihydroxy-6-[18F]-fluoro-L-phenylalanine (FDOPA) hypermetabolic ROls (green) are created from pre-operative images. Resected ROIs (red) are created from post-operative images, which have been registered to the pre-operative images. Residual contrastenhanced ROIs, residual FLAIR ROIs (blue), and residual FDOPA hypermetabolic ROIs (green) are created by subtracting resected ROIs from pre-surgery ROIs. In this slice, the contrast-enhanced ROI is completely resected. 